\title{
Optimal Time for Bile Duct Injury Reconstruction. Early Does Not Mean Better: a Multicenter Retrospective Analysis of a Hepato-pancreatic- biliary Group
}

Carlos Eduardo Rey Chaves ( $\square$ carlosrey991@gmail.com )

Hospital Universitario Mayor - Méderi - HPB Surgery Department Bogotá

Danny Conde Monroy

Hospital Universitario Mayor - Méderi - HPB Surgery Department Bogotá

P. Torres Gómez

Universidad el Rosario Bogotá

Andrea Recamán

Universidad el Rosario Bogotá

Manuel Pardo

Universidad el Rosario Bogotá

JC Sabogal

Hospital Universitario Mayor - Méderi - HPB Surgery Department Bogotá

\section{Research Article}

Keywords: Bile duct injury, Laparoscopic cholecystectomy, Outcome, Post-cholecystectomy,

Hepaticojejunostomy

Posted Date: March 8th, 2022

DOI: https://doi.org/10.21203/rs.3.rs-1344920/v1

License: (c) (i) This work is licensed under a Creative Commons Attribution 4.0 International License.

Read Full License 


\section{Abstract}

\section{Background}

Common bile duct injury it's a severe and complex complication, and it's related to increased rates of morbidity and mortality. Early recognition and on-time diagnosis plus multidisciplinary management led by a hepatobiliary surgeon show fewer complications rate and best postoperative outcomes. No guideline exists among the proper time of reconstruction. This study aims to describe the experience of a specialized Hepato-Pancreatic-Biliary (HPB) group and to analyze the outcomes regarding the time of bile duct injury (BDI) repair.

\section{Methods}

A multicenter retrospective review of a prospectively collected database was conducted. All the patients older than 18 years old that underwent common bile duct reconstruction between January 2014 December 2020 were included. Analysis and description of preoperative characteristics and postoperative outcomes were performed. A reconstruction time-based group differentiation was made and analyzed.

\section{Results}

37 patients underwent common bile duct reconstruction between January 2014 and December 2020. $62.16 \%$ of the patients were female. The mean age was 50.4 years $+/-19.5$ years. The most common injury was type E3 (29.73\%). Hepaticojejunostomy was performed in $75.68 \%$ of the patients. Delayed reconstruction (> 72 hours) was performed in the majority of the cases (81.08\%) due to delays in the referral centers. No statistically significant difference regarding complications in early or delayed BDI reconstruction. The mortality rate was $2.7 \%(n=1)$. 2-year follow-up bilioenteric stenosis was observed in 7 patients. Biloma showed a statistical relationship with complex bile duct injuries $(p=0.02)$.

\section{Conclusion}

Common bile duct injury remains to be a concerning complication in general surgery. The multidisciplinary approach shows a lesser complication rate. The time of BDI reconstruction seems not to be a significant factor related to complications. In this study, biloma was associated with complex bile duct injury, However, further studies are needed to confirm our results.

\section{Introduction}

700.000 cholecystectomies are performed annually in the United States [1-4] and laparoscopic cholecystectomy is currently the gold standard of symptomatic gallstones or acute cholecystitis management [1, 2]; Its benefits over open cholecystectomy are fast patient recovery, less postoperative pain, shorter hospital stay, and faster reintegration to daily activities, $[2,10,13,17]$. 
Although surgeons around the world have been accumulating experience and the learning curve has become steeper [17], in the last three decades there has been an increase in biliary tree lesions [11]. latrogenic biliary duct lesions can occur by either an open approach or by laparoscopy and it is associated with significant morbidity and low but not negligible mortality $[3-4,7]$.

Surgery by laparoscopy shows two to three times higher rates of bile duct lesions $(0,4 \%-0,6 \%)$ in comparison to an open approach $(0.2 \%-0.3 \%)[6,10,12-13]$. Patients who suffer common bile duct injury show increased 1 -year mortality of approximately $7.2 \%$, and $14,5 \%$ in a 5 -year follow-up s $<$ pan [13 $-4,19-22]$. Additionally, these lesions have an impact on the healthcare system, increasing costs up to $126 \%$ after bile duct repair. $[4-6,14-20]$

In order to decrease bile duct injuries, risks must be identified both previously and during surgery. A preoperative evaluation of risk factors, early injury identification, and accurate management of bile duct injuries are crucial to decrease morbidity and mortality. Also, it's well established the importance of the experience of the surgeon and formal Hepatobiliary and pancreatic (HPB) training when treating bile duct injuries. As Halle-Smith et al describe, reconstruction performed by a non-hepatobiliary surgeon increases a 4-time risk to present postoperative complications [21]. Among those relevant factors, time of reconstruction rises in literature as a key component of reduced morbidity and mortality [33]. Early reconstruction is associated with a shorter hospital stay, costs reduction, and reduced burden of the patient. On the contrary, delaying surgical reconstruction allows for optimization of the clinical condition, sepsis control, and delimitation of bile duct ischemia, eventually influencing long-term strictures and bile duct leak [33]. Some reports describe no relevance in the time of reconstruction for the early and delayed complication rates $[34,35]$. Therefore, this topic is still a matter of debate.

The current study aims to describe the experience in the management of common bile duct injury after laparoscopic/open cholecystectomy by a hepatobiliary group in high complexity centers in Colombia and to describe the association between time of reconstruction and short- and long-term complications.

\section{Methods}

The present study was performed in four centers where the iatrogenic biliary injury occurred (Two academic and 2 non-academic hospitals). A retrospective analysis was done. Biliary reconstruction was performed by the same HPB surgeon. Ethical compliance with the Helsinki Declaration, current legislation on research Res. 008430 - 1993 and Res. 2378 - 2008 (Colombia) and the International Committee of Medical Journal Editors (ICMJE) were ensured under the Ethics and Research Institutional Committee (IRB) approval. Institutional Review Board approval (Hospital Universitario Mayor Méderi, Universidad el Rosario ) from the institutions involved. Patients over 18 years old who were diagnosed with common bile duct injury following laparoscopic or open cholecystectomy between January 2014 and December 2020, were included.

Demographic characteristics, risk factors, remission time, intraoperative findings, confirmatory and paraclinical imaging studies performed, type of injury (Strasberg classification system), postoperative 
evolution, complications based on the Clavien-Dindo classification, and postoperative mortality were included. All patients were followed for postoperative stenosis at 6-12 and 24 months. Descriptive statistics of all study parameters were provided. Data were analyzed using STATA 17 software. Continuous data were summarized by their mean, median, standard deviation, and interquartile range. Categorical data were summarized by their frequency and proportion. Bivariate analysis was performed. Qualitative variables were analyzed using chi-square statistics (Fisher's exact test when appropriate). Quantitative variables were analyzed, based on normality, with Spearman's or Pearson's association's correlation coefficient accordingly. A multivariate analysis was performed to evaluate the association between clinical variables (Cholangitis, jaundice, and biloma), and the severity of the BDI.

Association among clinical variables and the severity of bile duct injury was evaluated. Complex injuries were defined as Strasberg types E3-E5. Clinical findings such as abdominal pain, cholangitis, biloma, and comorbidities (arterial hypertension and diabetes mellitus type 2) were evaluated. The significant statistical association was defined if a $p$-value $<$ or equal 0.05 was reached.

Timing of the reconstruction was defined in three groups: intraoperative, early ( $<72$ hours since the BDI) or late ( $>72$ hours). Associations between the timing of the reconstruction and postoperative outcomes such as mortality, bilio-enteric anastomotic stricture, ICU requirement, and Clavien Dindo classification were evaluated. The significant statistical association was defined if a p-value < or equal 0.05 was reached.

\section{Surgical approach}

Mederi Hospital is a referral center in HBP complex surgeries in Bogota. latrogenic biliary injuries were done in three other hospitals. However, the same HPB surgeon was able to perform reconstruction in all four institutions.

A standardized technique was performed. A midline supraumbilical laparotomy was created. Blunt dissection was performed by using low-energy electrocautery throw embryologic planes. The hepatoduodenal ligament was mobilized and adherences dissected. A Kocher maneuver was performed. Right after, Hilar plate dissection and identification of the common bile duct injury was done. Biloma was drained, clots and old bleeding were removed when needed. The abdominal cavity was cleaned. Healthy bile duct tissue was exposed. Standard transmesocolic one layer Roux-en-Y hepaticojejunostomy (HJ) was performed using either 4-0 or 5- 0 polydioxanone suture depending on bile duct thickness. Finally, intestinal transit is reconstructed by two layers of side-to-side jejunojejunostomy by using $4-0$ polydioxanone at 60 to $90 \mathrm{~cm}$ to the HJ. Either Jackson-pratt or Blake drain was left based on specific criteria and availability.

\section{Follow-up}

By institutional protocol, follow-up was performed 6, 12, 24 months to determine late postoperative complications, and postoperative bilio-enteric anastomosis stricture. Cholangio-resonance was 
performed to confirm the suspected diagnosis.

\section{Results}

37 patients were collected between Hospital Universitario Mayor Méderi (25) and Hospital Universitario San Ignacio (9); one patient from Clínica Palermo and two patients from Clínica Colsubsidio. Surgical management, follow up and data analysis was evaluated together for analysis.

\section{Demographic - Clinical}

A total of 37 patients were included in the study. The mean age was 50.4 years $+/-19.5$ years. Female constituted $62,16 \%(n=23)$ of the cases. Body Mass Index (BMI) average was a range between 18,5-24,9 $\mathrm{kg} / \mathrm{m} 2(70,27 \% \mathrm{n}=26)$. The initial surgical approach in the primary surgery was mainly laparoscopic $(51 \% n=19)$, and $21 \%(n=8)$ required conversion to laparotomy due to surgeon decision. Tokyo classification in cholecystitis patients was Tokyo I $(18,92 \%, n=7)$, Tokyo $2(54,05 \%, n=20)$, Tokyo 3 $(5,41 \%, n=2)$ and no cholecystitis $(21,62 \%, n=8)$. After biliary reconstruction, Blake drain was inserted in $54.04 \%(n=20)$ of the patients because of biliary leak after finishing reconstruction or previous biloma found. Significant number of patients were operated at teaching hospitals $(45,95 \%-n=17)$. Summarized characteristics are displayed in table 1. (See appendix)

\section{Diagnosis}

In our series, bile duct injury diagnosis was classified into three groups. During surgery $(40,54 \% / n=15)$, at first $72 \mathrm{~h}$ of the lesion $(3,41 \% / n=2)$ and, late diagnosis after $72 h(54,05 \% / n=20)$. In the first group, $76.19 \%$ of bile duct injury was found through intraoperative cholangiography. In the early and late group of patients, confirmation of the injury was made in the postoperative time by cholangio-resonance in $43,24 \%$ of the cases. $18,92 \%$ of the patients followed an Endoscopic retrograde cholangiopancreatography (ERCP) to diagnose the injury (See table 2 - Appendix). A routine blood test was made for all patients (total bilirubin, direct bilirubin, alkaline fosfatase, white blood cell count, serum creatinine y partial thromboplastin time). Results are described in Table 3. (See appendix)

\section{Type of injury}

The type of injury was classified according to the Strasberg classification. The most frequent lesion was E2 $(29.73 \%, n=11) \cdot 67,56 \%$ of patients had complex biliary tree bifurcation injury (E 3-4), (See graphic 1). In addition, 2 patients were documented with arterial injury, in both cases with right hepatic artery ligation. However, incomplete data was found. No portal injury was documented.

there is not enough information on the surgical report about vascular injuries.

\section{Timing of the reconstruction}

Biliary reconstruction was performed in most of the cases $(81.08 \%, n=30)$ in a range between 72 and 120 hours after the diagnosis. Two particular cases were reconstructed after a month. One of them due to 
sepsis and the other one due to administrative delays to referring the patient. Four patients were taken for reconstruction between 24 to 72 hours after diagnosis and only three cases had reconstructive surgery in the same surgical time as cholecystectomy; those occurred in our base center after the general surgeon recognized the injury. Delayed reconstruction in our study $(>72 h)$ shows no statistical relationship with mortality, bile duct stricture, or severe postoperative complications defined as Clavien Dindo III - IVa ( $p=$ $0.1 ; p=0.07 ; p=0.45 ; p=0.56$ respectively), with no differences between this approach, and surgical reconstruction in the early post-cholecystectomy period. (See table 5 - Appendix).

\section{Surgical reconstruction}

The early recognition of the lesion was a relevant factor to adequately perform a bile drain and prompt referral to HPB service. In our analysis $75.68 \%(n=28)$ reconstruction was done with hepaticojejunostomy, $5.41 \%(n=2)$ underwent choledocoplasty, and 2 patients $(5.41 \%)$ were managed with primary closure of the bile duct. One patient $(2,70 \%)$ required a double bilioenteric anastomosis. Intraoperative bleeding was on average $244 \mathrm{cc}$. The average surgical time was 206.83 minutes. In the first surgery, a drain was left in $45,95 \%$ of the patients with suspected bile duct injury, It is important to point out that patients should have bile duct drains with a stent in situ during surgical injury if it is recognized or subhepatic drains should be placed to avoid biloma or peritonitis. This is a key factor when it is mandated to refer patients to an HPB center.

\section{Postoperative results}

Intensive care unit (ICU) was required in seven cases after surgery period due to cholangitis and mean ICU stay there were 0.72 days $+/-1.62$. Postoperative complications were analyzed using the Clavien-Dindo classification. Type 1 represents the majority of our patients with $70.27 \%(n=26)$, followed by type $3 B$ occurring in 6 patients $(16.22 \%)$, and type 2 with $8.11 \%(n=3)$ of the patients who need a blood transfusion. Types $3 \mathrm{~A}-4 \mathrm{~A}$ each occurred in 1 patient $(2.70 \%)$, and only one patient $(2.70 \%)$ required surgical re-intervention to address its complication. No patients showed complications of type 4B. Mortality was present in one case $(2.7 \%)$, this particular case was reconstructed one month after bile duct injury due to a delay in biliary injury recognition and delay in referral to our HPB center. Bilio-enteric stricture was observed in 7 patients after a 2-year follow-up and all of these cases required new bilioenteric reconstruction.

Biloma has a statistical relationship with complex common bile duct injury ( $p=0.02, \mathrm{Cl} 95 \%)$, the presence of biloma shows a 41-fold risk of presenting with a Type E3-E4 Strassberg injury of the bile duct. (See table 4 - Appendix)

\section{Discussion}

Since the first description of laparoscopic cholecystectomy in 1985, it has become the gold standard in care for patients with gallstones and acute cholecystitis. Despite the decrease in complications due to a steeper learning curve, the incidence of bile duct injury continues to be one of the most feared 
complications, currently occurring at 0.2 to $0.7 \%$ of patients undergoing surgery [10]. The early diagnosis and optimal treatment are the cornerstones to prevent bad outcomes or major complications [10].

A controversial debate on the optimal timing of $\mathrm{HJ}$ for BDI still persists in the literature. A wide variety of cut-off times for "early" and "delayed" surgery has been described and no guidelines at present show the proper time of BDI repair [34]. However, early reconstruction is always recommended in HPB centers around the world. In this study, we used the most common classification as early (first 72 hours of reconstruction after the BDI diagnosis) and delayed (after 72 hours) [35]. In this study, no associations in complications rates were found between those cut-off values, and the complication rate was similar in those groups (Intraoperative Group $p=0.1$, Early group $p=0.6$, delayed group $p=0.5$ ). Some recent reports support this finding, a collaborative retrospective study from the European-African HPB Association concluded that the timing of reconstruction did not have an impact on severe postoperative complications, reinterventions, or mortality [36]. As well, Stewart and Way found in a multivariate analysis that the time of reconstruction was not an important factor [37]. However, Schreuder et al show that reconstruction between 2-6 weeks has an increased rate of postoperative morbidity and hepaticojejunostomy stricture [33]. Therefore, based on our data, the timing of reconstruction it's not related to increased postoperative complications, mortality, or anastomotic stricture, and suggests that early does not mean better in BDI reconstruction, however, a delay of more than 4 weeks could be detrimental in postoperative outcomes[33, 36].

Several risk factors must be revised in the preoperative time to classify the risk of potential complications. There are well-described factors in literature such as acute cholecystitis and cholangitis that were associated with more complex injuries (Type E) with statistically significant value [30]. In this group, there was a higher rate of biliary duct lesions in patients with acute cholecystitis; $78.3 \%$ of the cases had acute cholecystitis at the time of the injury. However, it was not clearly associated with the complexity of the CBD injury $(p=0.06)$. Reasons given for this association may be related to the degree of inflammation, the development of fibrosis, and adhesion processes, which hinder the adequate exposure of the surgical field and provide a modification in the anatomy that prevents an adequate identification of the structures that need surgical repair [27].

When occurred, identification of the bile duct injury is still an enormous concern, only one-quarter of injuries are recognized during surgery [28]. In this case series, $40,54 \%$ of cases were identified in the intraoperative time. Most of the lesions were identified in the postoperative time, the presence of symptoms such as fever, postoperative jaundice, and unmanageable abdominal pain should alert the possibility of bile duct injury. [17]. In this study, the most common symptom was unmanageable postoperative pain in $70.27 \%$ of the patients. Jaundice appeared in $67.5 \%$ of cases and only $21.62 \%$ of patients presented with cholangitis [17].

The experience of a hepatobiliary surgeon and a specialized group has proven to be of relevance to ensure optimal results. $75 \%$ of surgeons attempt to repair the injury on their own with a poor success rate of $17 \%[10]$. The impact of bile duct injuries is high, this generates an increase in hospital stay and care 
costs. Also, the emotional toll of this complication is high as any health worker who has been in relation to a case with bile duct injury clearly knows the emotional value that each case represents, not only for the patient and their family but also for all the nursing staff and the surgical team. [37]. Therefore, based on a significant amount of publications, the international organizations for HPB surgeons (AHPBA, IHPBA) state that bile duct reconstruction must be performed only by HPB surgeons.

Long-term follow-up data for this type of lesions is scarce given the low frequency of presentation and the low publication of case series in our setting [14]. In a series of 400,000 cholecystectomies, Sinha et al. found that biliary tree injuries had a 6 -fold increased risk of mortality at 1 year compared to those without bile duct lesions [18]. One of the most frequent complications in long-term follow-up is anastomotic stenosis. In our series, 7 patients presented biliary stricture in long-term follow-up established at two years.

The presence of biloma in early or late BDI detection showed statistical association with severity of the injury ( $p$ 0.02), with a 41.7 increased risk-fold to present highly complex common bile duct injuries defined in this study as type E3-5 Strasberg lesions.

Vascular compromise is a well-described characteristic associated with complications, however in our patients, there were only 2 cases with confirmed vascular compromise, this finding can be associated with an underreported description in the involved hospital.

Strengths identified in our study are management by the same hepatobiliary group in multiple institutions and the possibility of follow-up at 30 days and 24 months postoperatively. Results in this study should be interpreted in light of several limitations. Only surgical nature of patients undergoing reconstruction as we did not have data on the total incidence of bile duct lesions (lesions with endoscopic management or patients who died before reoperation), as well as the retrospective nature of the study and the small number of patients included. Finally, a possible underreported description of associated complications like the vascular compromise could influence the results.

\section{Conclusion}

Bile duct injury is a severe and complex postoperative complication that increases morbidity and mortality rates in the short and long term in patients undergoing cholecystectomy. Based on our results the time of reconstruction it's not related to early and late complications. However, it is recommended to repair as soon as the clinical condition of the patient allows.

\section{Declarations}

\section{Compliance with Ethical Standards:}

\section{Funding:}


This study does not receive any funding.

\section{Conflict of Interest:}

Authors do not have any conflict of interest.

\section{Ethical approval:}

All procedures performed in studies involving human participants were in accordance with the ethical standards of the institutional and/or national research committee (Hospital Universitario Mayor Méderi Universidad el Rosario ) and with the 1964 Helsinki declaration and its later amendments or comparable ethical standards.

\section{Informed consent:}

Informed consent was obtained from all individual participants included in the study, and reposes in clinical history

\section{Availability of Data and Materials}

The datasets used and/or analysed during the current study available from the corresponding author on reasonable request due to data protection protocol of the institution.

\section{References}

1. Wernberg, Jessica A., and Dustin D. Lucarelli. "Gallbladder cancer." Surgical Clinics of North America 94.2 (2014): 343-360.

2. Siegel R, Naishadham D, Jemal A. Cancer statistics, 2013. CA Cancer J Clin 2013;63:11-30.

3. Moore KL, Dalley AF. Clinically oriented anatomy. $5^{\text {th }}$ edition. Baltimore (MD): Lippincott Williams \& Wilkins; 2006.

4. Halbert C, Altieri MS, Yang J, et al. Long-term outcomes of patients with common bile duct injury following laparoscopic cholecystectomy. Surg Endosc 2016;30(10):4294-9.

5. Netter FH. Atlas of human anatomy. In: Hansen JT, Benninger B, Brueckner JK, et al, editors. Atlas of human anatomy. $5^{\text {th }}$ edition. Philadelphia: Elsevier inc; 2011. P. 240-330.

6. Ismael HN, Cox S, Cooper A, et al. The morbidity and mortality of hepaticojejunostomies for complex bile duct injuries: a multi-institutional analysis of risk factors and outcomes using NSQIP. HPB (Oxford) 2017;19(4):352-8. 
7. Mercado MA, Chan C, Orozco H, Tielve M, Hinojosa CA. Acute bile duct injury. The need for a high repair. Surg Endosc. 2003;17:1351-5

8. Traverso LW. Carl Langenbuch and the first cholecystectomy. Am J Surg. 1976;132:81-2.

9. Muhe E. Laparoscopic cholecystectomy-late results. Langenbecks Arch Chir Suppl Kongressbd. 1991:416-23.

10. Sicklick JK, Camp MS, Lillemoe KD, Melton GB, Yeo CJ, Campbell KA, et al. Surgical management of bile duct injuries sustained during laparoscopic cholecystectomy: perioperative results in 200 patients. Ann Surg. 2005;241: 786-92.

11. Dixon JA, Morgan KA, Adams DB. Management of common bile duct injury during partial gastrectomy. Am Surg 2009;75(3):719-21.

12. Fragulidis G, Marinis A, Polydorou A, et al. Managing injuries of hepatic duct confluence variants after major hepatobiliary surgery: an algorithmic approach. World J Gastroenterol 2008;14(19):3049

13. Fong ZV, Pitt HA, Strasberg SM, et al. Diminished survival in patients with bile leak and ductal injuries: management strategy and outcomes. J Am Coll Surg 2018; 226(4):568-76.e.

14. Barrett M, Asbun HJ, Chien H-L, et al. Bile duct injury and morbidity following cholecystectomy: a need for improvement. Surg Endosc 2017;32(4):1683-8.

15. Conrad C, Wakabayashi G, Asbun HJ, et al. IRCAD recommendation on safe laparoscopic cholecystectomy. J Hepatobiliary Pancreat Sci 2017;24(11):603-15.

16. Pucher PH, Brunt LM, Fanelli RD, et al. SAGES expert Delphi consensus: critical factors for safe surgical practice in laparoscopic cholecystectomy. Surg Endosc 2015;29(11):3074-8.

17. Bobkiewicz A, Krokowicz q, Banasiewicz T, et al. latrogenic bile duct injury. A significant surgical problem. Assessment of treatment outcomes in the department sown material. Pol Przegl Chir 2015;86(12):576-83.

18. Sinha S, Hofman D, Stoker DL, et al. Epidemiological study of provision of cholecystectomy in England from 2000 to 2009: retrospective analysis of hospital episode statistics. Surg Endosc 2012;27(1):162-75.

19. Kohn JF, Trenk A, Kuchta K, et al. Characterization of common bile duct injury after laparoscopic cholecystectomy in a high-volume hospital system. Surg Endosc 2018;32(3):1184-91.

20. Tornqvist B, Stromberg C, Persson G, et al. Effect of intended intraoperative cholangiography and early detection of bile duct injury on survival after cholecystectomy: population based cohort study. BMJ 2012;345(1):1-10

21. Halle-Smith JM, Hodson J, Stevens LG, Dasari B, Marudanayagam R, Perera T, Sutcliffe RP, Muiesan $\mathrm{P}$, Isaac J, Mirza DF, Roberts KJ. A comprehensive evaluation of the long-term economic impact of major bile duct injury. HPB (Oxford). 2019 Oct;21(10):1312-1321. Doi: 10.1016/j.hpb.2019.01.018. Epub 2019 Mar 9. PMID: 30862441.

22. Kholdebarin R, Boetto J, Harnish JL, et al. Risk factors for bile duct injury during laparoscopic cholecystectomy: a case-control study. Surg Innov 2008;15(2):114-9. 
23. Jab1onska B, Lampe P. latrogenic bile duct injuries: etiology, diagnosis and management. World $\mathrm{J}$ Gastroenterol 2009;15(33):4097.

24. Gómez FR, Ramia JA, et al. Lesiones iatrogénicas de la vía biliar. Cirugía spañola, Cir Esp 2010;88:211-21 doi:10.1016/j.ciresp.2010.03.045

25. Kerin MJ, Gorey TF. Biliary injuries in the laparoscopic era. Eur J Surg. 1994;160:195-201.

26. Fischer CP, Fahy BN, Aloia TA, et al. Timing of referral impacts surgical outcomes in patients undergoing repair of bile duct injuries. HPB (Oxford) 2009;11(1):32-7.

27. Waage $A$, Nilsson M. latrogenic bile duct injury: a population-based study of 152776 cholecystectomies in the Swedish Inpatient Registry. Arch Surg. 2006;141:1207-13.

28. Karvonen J, Gullichsen R, Laine S, Salminen P, Gronroos JM. Bile duct injuries during laparoscopic cholecystectomy: primary and long-term results from a single institution. Surg Endosc. 2007;21(7):1069-73.

29. Fullum TM, Downing SR, Ortega G, et al. Is laparoscopy a risk factor for bile duct injury during cholecystectomy?. JSLS. 2013;17(3):365-370. Doi:10.4293/108680813X13654754535638.

30. Grönroos JM, Hämäläinen MT, Karvonen J, Gullichsen R, Laine S. Is male gender a risk factor for bile duct injury during laparoscopic cholecystectomy?. Langenbecks Arch Surg. 2003;388(4):261-264. Doi:10.1007/s00423-003-0407-1

31. Stewart L. latrogenic biliary injuries: identification, classification, and management. Surg Clin North Am. 2014;94(2):297-310

32. Schol FP, Go PM, Gouma DJ. Risk factors for bile duct injury in laparoscopic cholecystectomy: analysis of 49 cases. Br J Surg. 1994 Dec;81(12):1786-8. Doi: 10.1002/bjs.1800811225. PMID: 7827940.

33. Schreuder AM, Nunez Vas BC, Booij KAC, van Dieren S, Besselink MG, Busch OR, van Gulik TM. Optimal timing for surgical reconstruction of bile duct injury: meta-analysis. BJS Open. 2020 Oct;4(5):776-786. Doi: 10.1002/bjs5.50321. Epub 2020 Aug 27. PMID: 32852893; PMCID: PMC7528508.

34. El Nakeeb A, Sultan A, Ezzat H, Attia M, Abd ElWahab M, Kayed T, Hassanen A, AlMalki A, Alqarni A, Mohammed MM. Impact of referral pattern and timing of repair on surgical outcome after reconstruction of post-cholecystectomy bile duct injury: A multicenter study. Hepatobiliary Pancreat Dis Int. 2021 Feb;20(1):53-60. Doi: 10.1016/j.hbpd.2020.10.001. Epub 2020 Oct 14. PMID: 33268245.

35. Nuzzo G, Giuliante F, Giovannini I, Murazio M, D'Acapito F, Ardito F, Vellone M, Gauzolino R, Costamagna G, Di Stasi C. Advantages of multidisciplinary management of bile duct injuries occurring during cholecystectomy. Am J Surg. 2008 Jun;195(6):763-9. doi: 10.1016/j.amjsurg.2007.05.046. PMID: 18367147.

36. A European-African HepatoPancreatoBiliary Association (E-AHPBA) Research Collaborative Study management group; other members of the European-African HepatoPancreatoBiliary Association 
Research Collaborative. Post cholecystectomy bile duct injury: early, intermediate or late repair with hepaticojejunostomy - an E-AHPBA multi-center study. HPB (Oxford) 2019; 21: 1641-1647.

37. Stewart L, Way LW. Laparoscopic bile duct injuries: timing of surgical repair does not influence success rate. A multivariate analysis of factors influencing surgical outcomes. HPB (Oxford). 2009 Sep;11(6):516-22. Doi: 10.1111/j.1477-2574.2009.00096.x. PMID: 19816617; PMCID: PMC2756640.

\section{Figures}

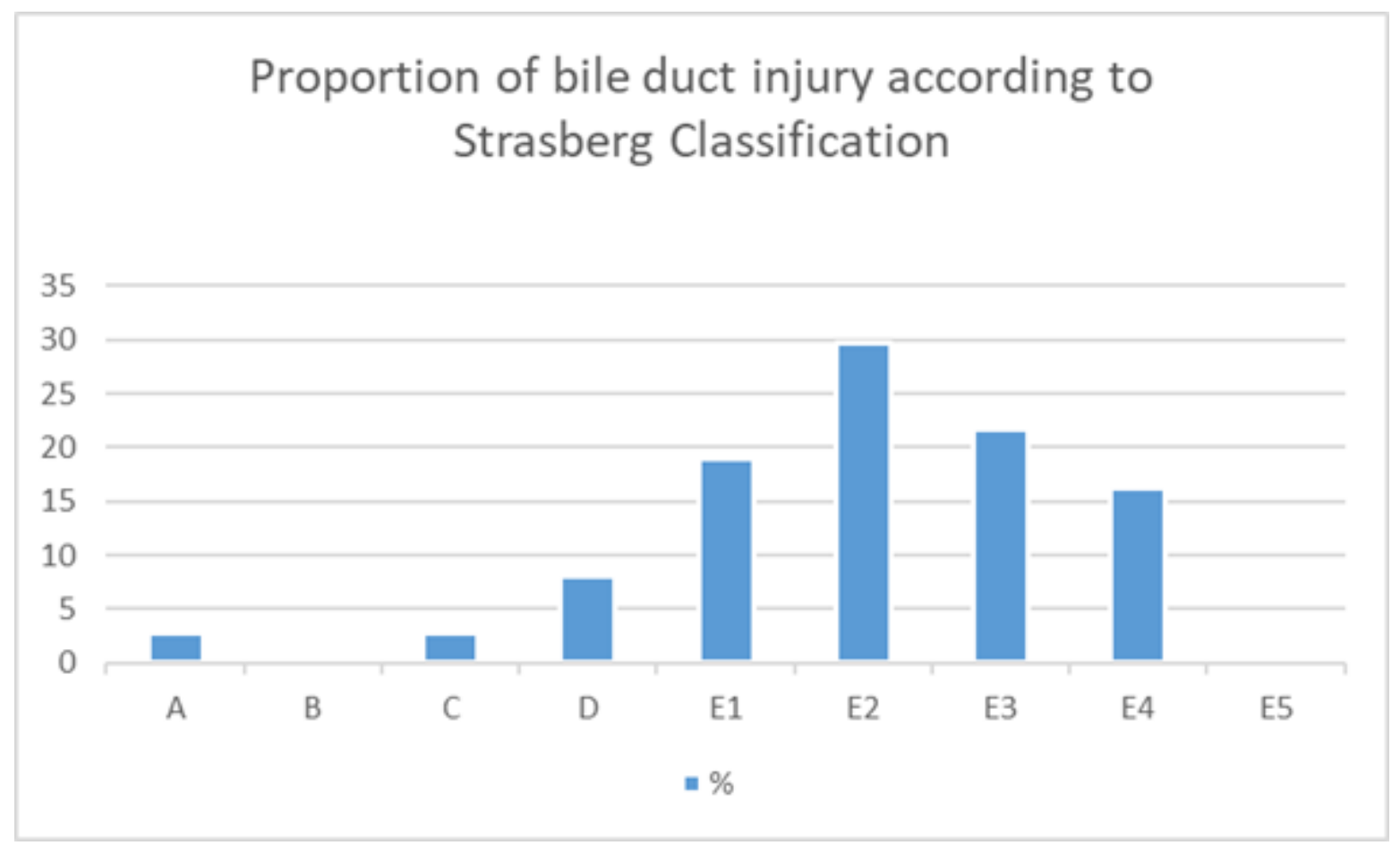

Figure 1

Graph 1 . Proportion of bile duct injury according to Strasberg Classification .

\section{Supplementary Files}

This is a list of supplementary files associated with this preprint. Click to download.

- AppendixBDI.docx

- DATABASEBDI.xIsx 